EPJ Web of Conferences 63, 01010 (2013)

DOI: $10.1051 /$ epjconf/ 20136301010

(C) Owned by the authors, published by EDP Sciences, 2013

\title{
The SPICE Detector at ISAC
}

\author{
A.B. Garnsworthy ${ }^{1}$ a , M. Moukaddam ${ }^{1}$, C. Bolton ${ }^{1}$, S. Ketelhut ${ }^{1}$, L.J. Evitts ${ }^{1}$, C. Andreoiu², \\ M. Constable ${ }^{1}$, G. Hackman ${ }^{1}$, R. Henderson ${ }^{1}$, and C.E. Svensson ${ }^{3}$ \\ ${ }^{1}$ TRIUMF, 4004 Wesbrook Mall, Vancouver, B.C., V6T 2A3, Canada \\ ${ }^{2}$ Department of Chemistry, Simon Fraser University, Burnaby, \\ British Columbia, Canada, V5A 1 S6 \\ ${ }^{3}$ Department of Physics, University of Guelph, Guelph, Ontario, Canada, N1G 2W1
}

\begin{abstract}
A new ancillary detector system for the TIGRESS HPGe array called SPectrometer for Internal Conversion Electrons (SPICE) is currently under development. SPICE consists of a segmented electron detector, photon shield and a permanent magnetic lens. SPICE will enable in-beam electron spectroscopy and, in coupling to the TIGRESS HPGe array, coincident gamma-electron spectroscopy with stable and radioactive beams.
\end{abstract}

\section{Introduction}

The nuclear shell model has been a cornerstone for our understanding of nuclear structure since its inception in 1955 [1, 2]. It describes very well a large number of the excitations in nuclei around spherical closed shells. A key question for nuclear structure is to understand the microscopic mechanisms responsible for the onset of deformation and collective excitations which are more difficult to describe in the traditional shell model approach. The evolution in nuclear structure between these two scenarios, particle-excitations of a spherical nucleus and collective excitations of a deformed nucleus, often takes place with the addition or removal of many pairs of valence nucleons to a doubly closedshell core. However, in almost all nuclei near shell closures, deformation and collective excitations can also occur simply with the addition of a few $\mathrm{MeV}$ of excitation energy. This is most apparent in nuclei identified as displaying shape coexistence close to the ground state [3].

One core feature of shape coexistence is understanding the nature and origin of low-lying $0^{+}$ states and the excitations built upon them. This can be challenging for two main reasons; firstly the electric quadrupole transitions de-exciting $2^{+}$states will have a larger branching ratio to the $0^{+}$ ground state simply because of the larger transition energy (even when the $\mathrm{B}(E 2)$ value is much larger to an excited $0^{+}$state), secondly all the experimental observables such as excitation energy, branching ratios and quadrupole transition strengths can be modified by quantum mechanical mixing with other configurations. This often clouds the true nature of the $0^{+}$band-head state and indeed the assignment of structures built upon it.

SPICE (SPectrometer for Internal Conversion Electrons) is a new device being constructed at TRIUMF-ISAC to perform in-beam electron spectroscopy with stable and radioactive ion beams. The device will enable the measurement of electric monopole transitions in exotic nuclei. Electric

a e-mail: garns@triumf.ca 

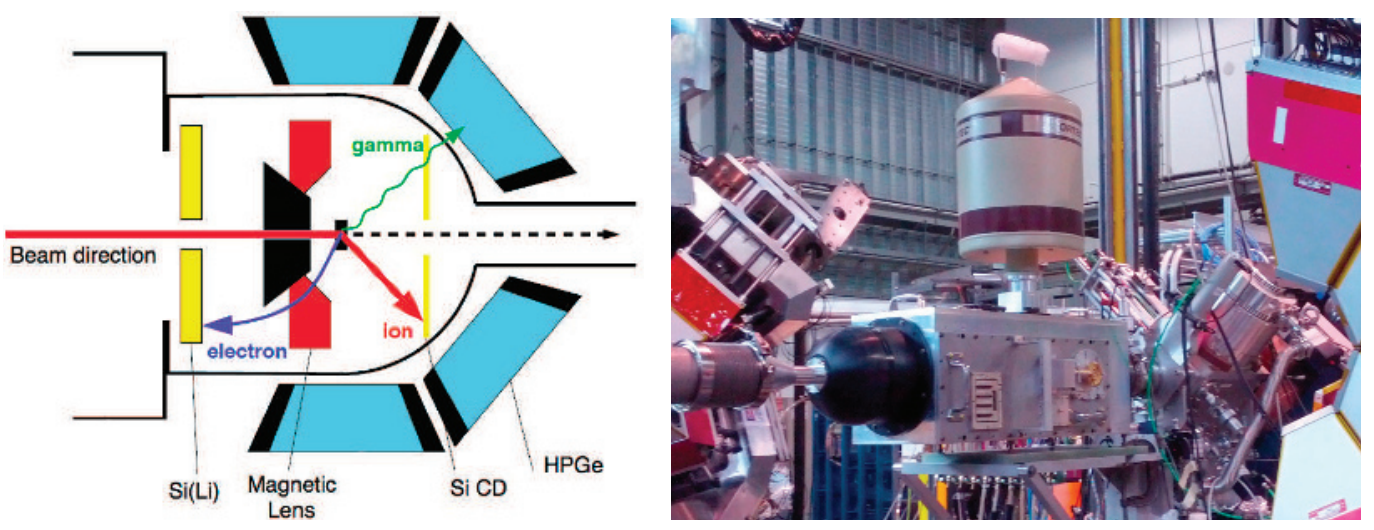

Figure 1. Left: Schematic view of the major components of SPICE. Right: Photograph of the SPICE chamber mounted inside TIGRESS.

monopole transition strengths can assist in the unravelling of the interpretation of the observed experimental data. Assuming that two states of the same spin and parity have a well defined deformation, the electric monopole strength of a transition between these states is directly proportional to the difference in their mean-squared charge radii and the degree of mixing between the two configurations.

SPICE will enable the identification of excited $0^{+}$states in exotic nuclei and the determination of electric monopole transition strengths which will help develop our understanding of shape coexistence away from the line of stability. In the case where shape coexistence develops as particle-hole excitations around a shell closure, these measurements will help address issues such as shell evolution as a function of isospin as well as the mechanisms driving the onset of collectivity and deformation in atomic nuclei.

\section{SPectrometer for Internal Conversion Electrons}

SPICE consists of an electron detector, photon shield and a magnetic lens as can be seen in the left of figure 1. The electron detector is an annular $6.1 \mathrm{~mm}$ thick lithium-drifted silicon detector with a diameter of $10 \mathrm{~cm}$. The detector is segmented into 120 individual segments arranged with 12 azimuthal sectors and 10 rings. The electron detector is located $11.5 \mathrm{~cm}$ upstream of the target and has a $10 \mathrm{~mm}$ hole at the centre for the heavy ion beam to pass through. A multi-layer (Tantalum steel, tin, copper) photon shield is positioned between the electron detector and the target to shield from direct irradiation by $\mathrm{x}$ rays and gamma rays. The magnetic lens collects and directs electrons emitted from the target around the photon shield to the electron detector. A silicon CD detector will detect the presence, direction and energy of the coincident recoiling heavy ion.

The magnetic lens consists of four magnetic sub-assemblies arranged around the photon shield. Each sub-assembly aligns with the suppression shield detectors of the TIGRESS HPGe Clover detectors in order to have as low an impact as possible on the quality of gamma-ray spectra. The trajectories of electrons through the lens is dependant on the strength and shape of the magnetic field. The highest grade Neodymium Iron Boron $(\mathrm{NdFeB})$ rare-earth permanent magnet is employed and the geometry of the magnets can be varied in many ways; thickness, length, cut-off angle. Through a detailed design process an optimal combination of these parameters was established which allows simple modification for different energy ranges. The magnetic lens and detector combination offers an absolute efficiency 
greater than $15 \%$ which exceeds the natural solid angle coverage of the detector. Three lens designs have been developed to cover the energy range from $100 \mathrm{keV}$ to $4 \mathrm{MeV}$.

\section{Status and Outlook}

Detailed designs and GEANT4 simulations of the spectrometer have been established. The components of the spectrometer have been fabricated and are undergoing assembly and testing at TRIUMF (figure 1). A detailed characterization of the detector and lens performance will be carried out in a bench-top setup using radioactive sources. Initial in-beam testing is anticipated for the fall of 2013 before first experiments using TIGRESS in conjunction with SPICE in 2014.

\section{Acknowledgements}

The SPICE collaboration would like to thank SEMIKON Detector GmbH, Karl-Heinz-Beckurts-Str. 13, 52428 Jülich, Germany (e-mail: info@ semikondetector.de, internet: www.semikon-detector.de) for developing the segmented $\mathrm{Si}(\mathrm{Li})$ detector. Construction of SPICE has been funded by the Canada Foundation for Innovation and the Ontario Ministry of Research and Innovation.

\section{References}

[1] M.G. Mayer and J.H.D. Jensen, Elementary Theory of Nuclear Structure (Wiley, New York, 1955)

[2] B.A. Brown and B.H. Wildenthal, Ann. Rev. Nucl. Part. Sci. 38, 29 (1988)

[3] K. Heyde and J.L. Wood, Rev. Mod. Phys. 83, 1467 (2011) 